\title{
An Experimental investigation of jet noise from septa(e) nozzles
}

Khairul Zaman, James Bridges, Amy Fagan and Cliff Brown NASA GRC, Cleveland, OH 44135

$2^{\text {nd }}$ AIAA/CEAS Aeroacoustics Conference

Lyon, France, May - June 2016

Supported by

Advanced Air Transport Technology (AATT) Project

Advanced Air Vehicles Program 


\title{
Outline of talk:
}

\author{
Introduction
}

\section{Experimental Facility}

Results and Discussion

\section{Summary}




\section{Distributed Propulsion}

(From Felder, Kim \& Brown 2009)

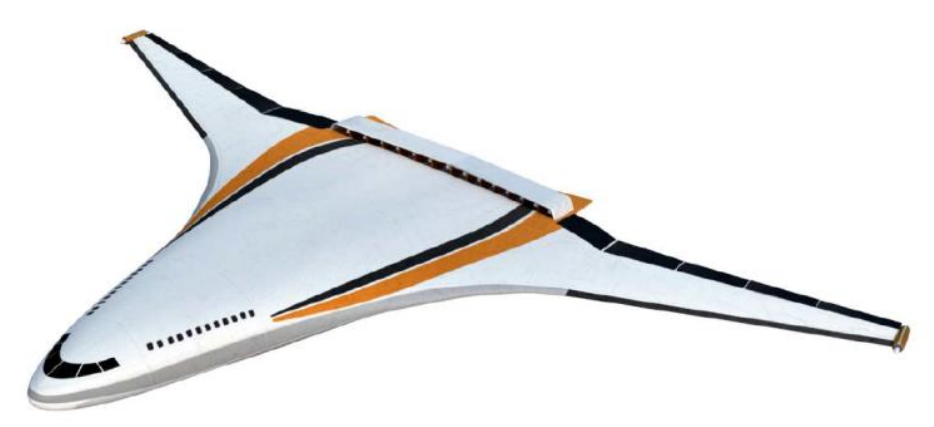

In one (hybrid) version of the concept each septum is driven by an electric fan

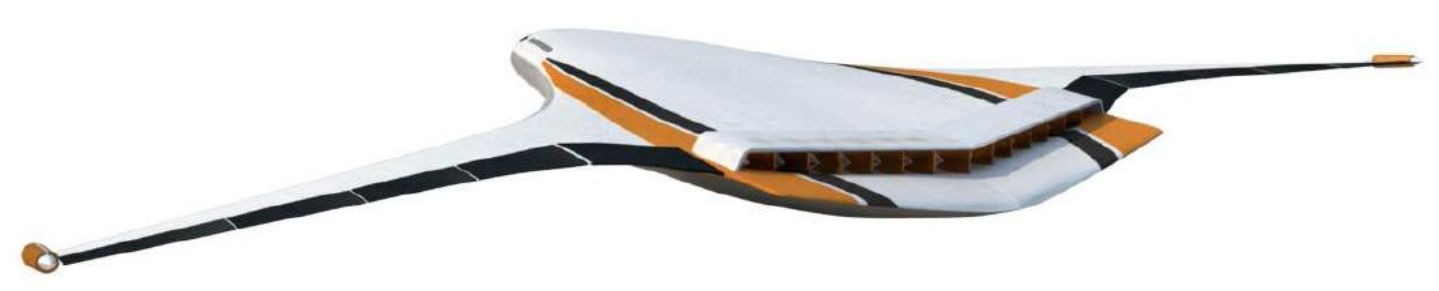

Concern about impact on noise. Will noise be greater than that from a equivalent single jet? 


\section{Experimental Facility}

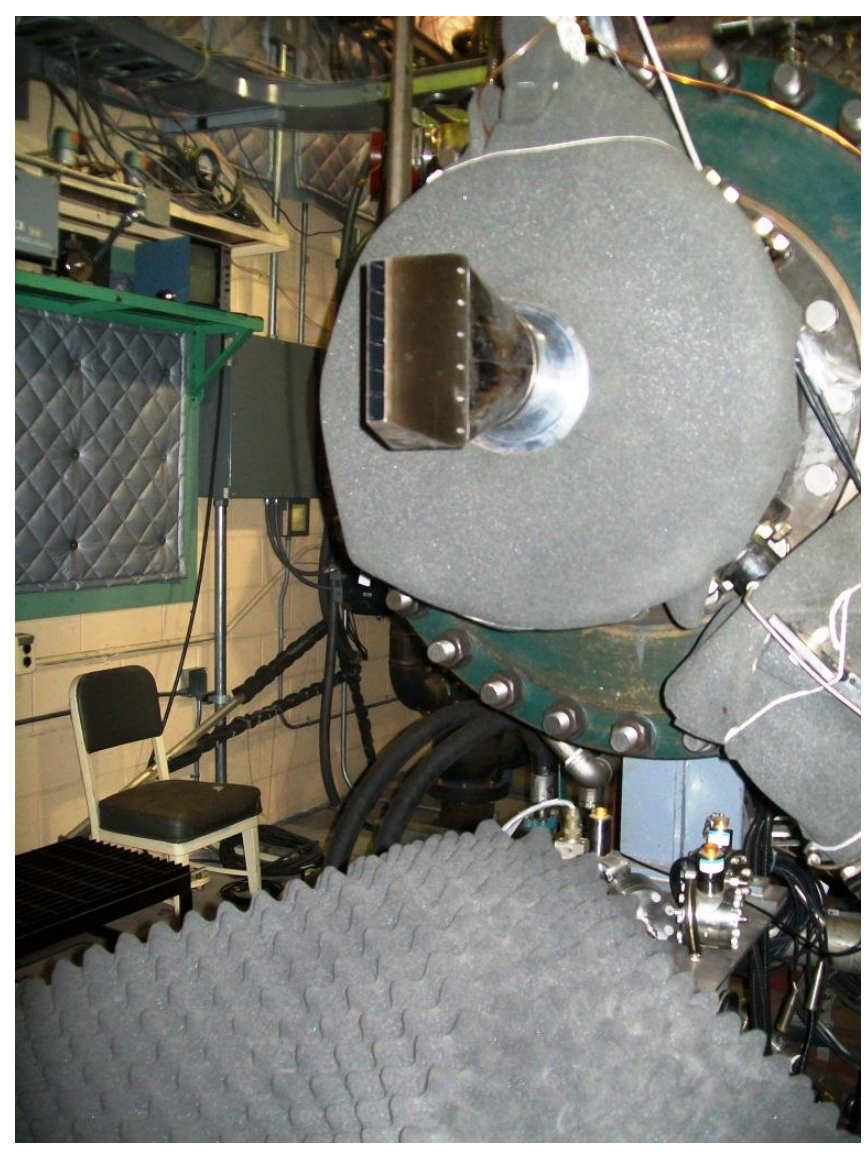

Open Jet rig (CW17)

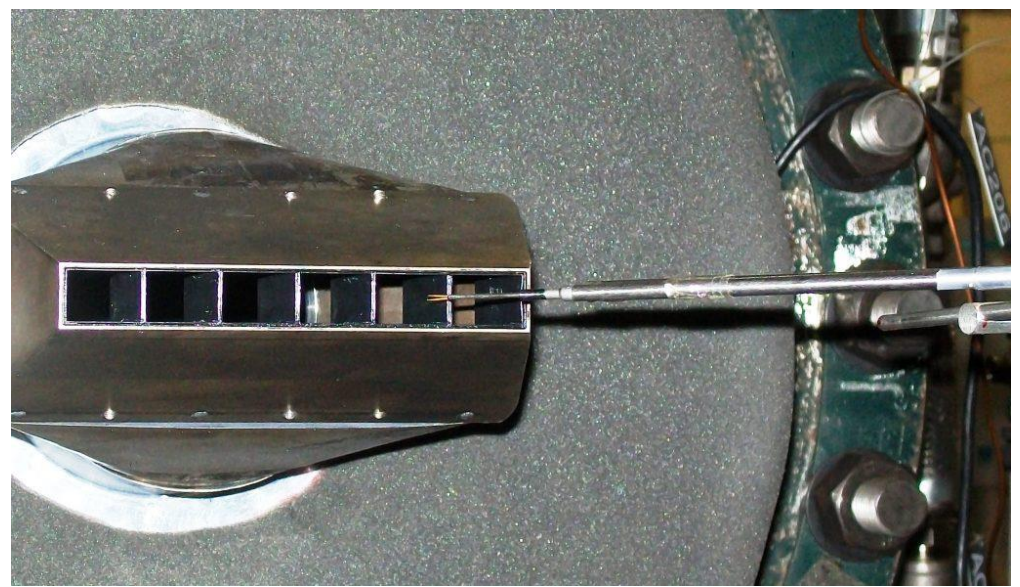

Close-up view of nozzle and HW

- Up to about $500 \mathrm{kPa}$ allowed

- Microphones overhead

- 8:1 rectangular nozzle $(14.1 \mathrm{~cm} \times 1.68 \mathrm{~cm})$

- Inserts made by 3-D printing 


\section{Experimental Facility (inserts)}

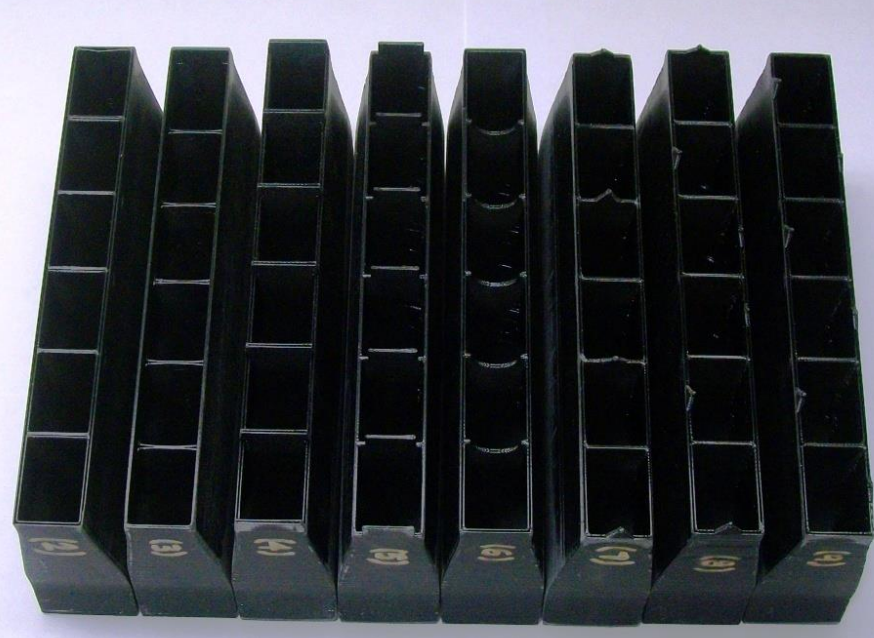

Picture of 8 inserts

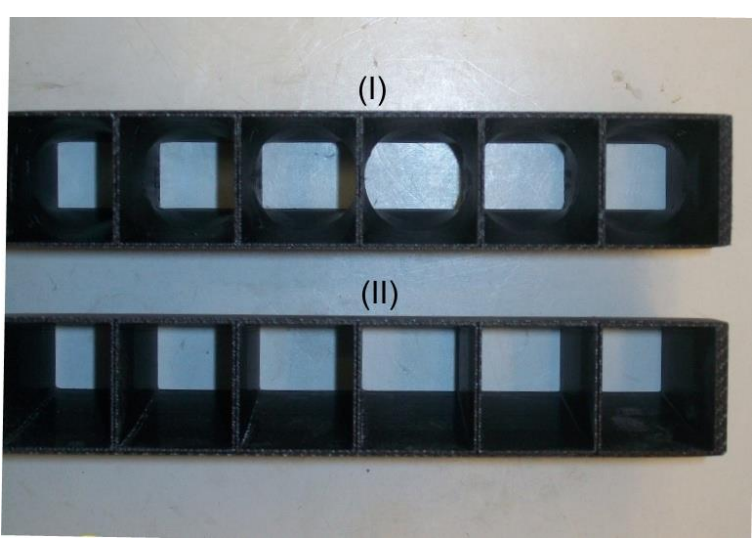

Internal geometry design I and design II
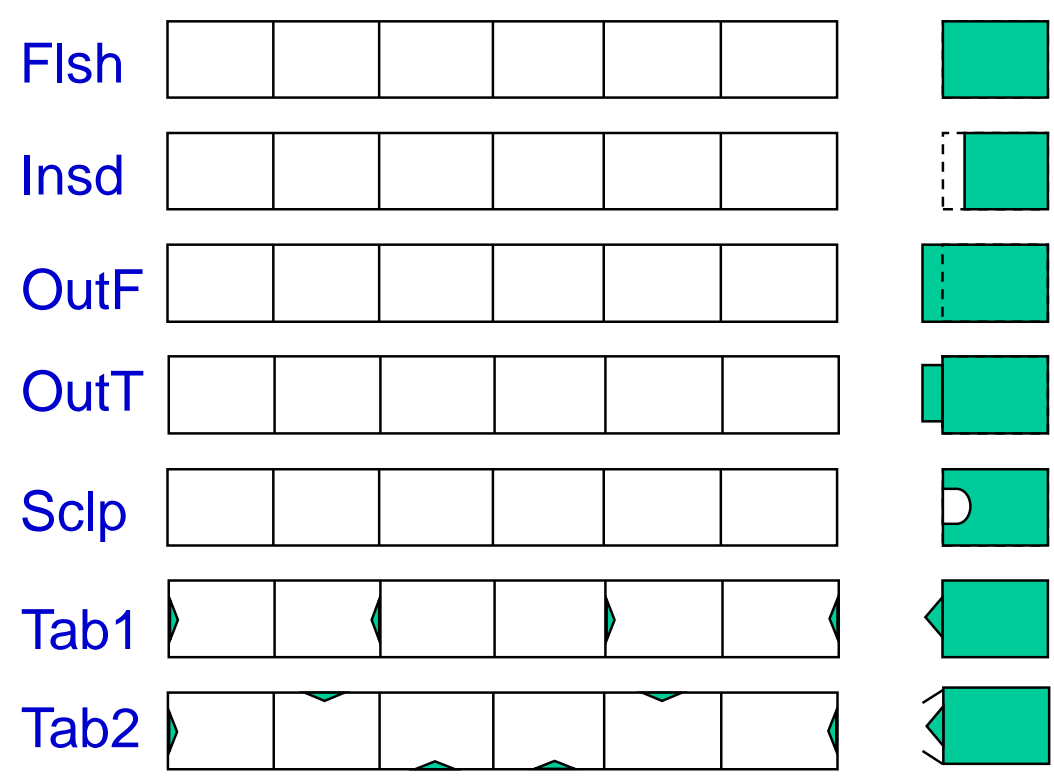

Schematic of exit shapes

-Different exit shapes examined for maximum noise reduction

-Number of septa varied with Design II 


\section{SPL Spectra comparison: Baseline vs. Flsh cases}
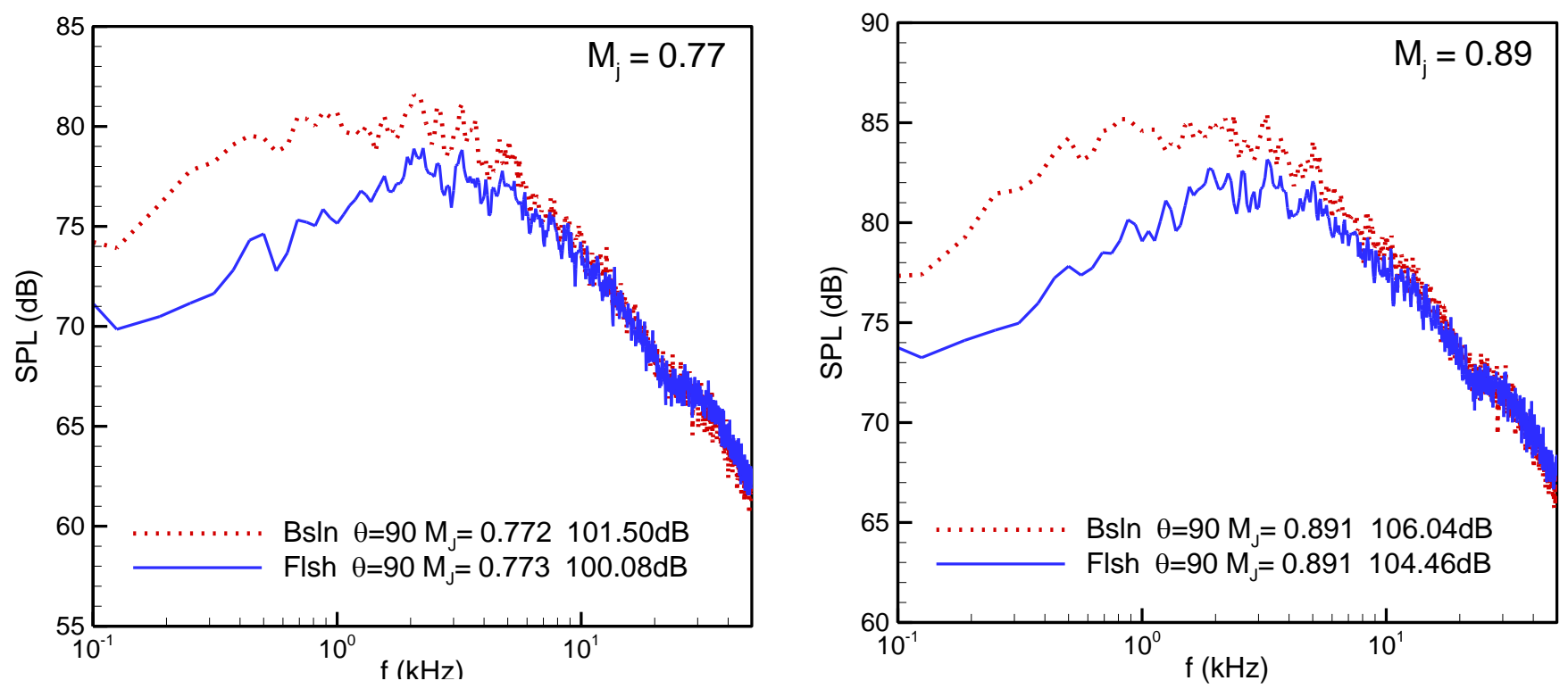

Lower noise on low frequency end for the Flsh case, at all $M_{j \text {. }}$

Not accounted for by exit area reduction $\left(11 \%\right.$ smaller $D_{\text {eq }}=>$ $1.3 \mathrm{~dB})$
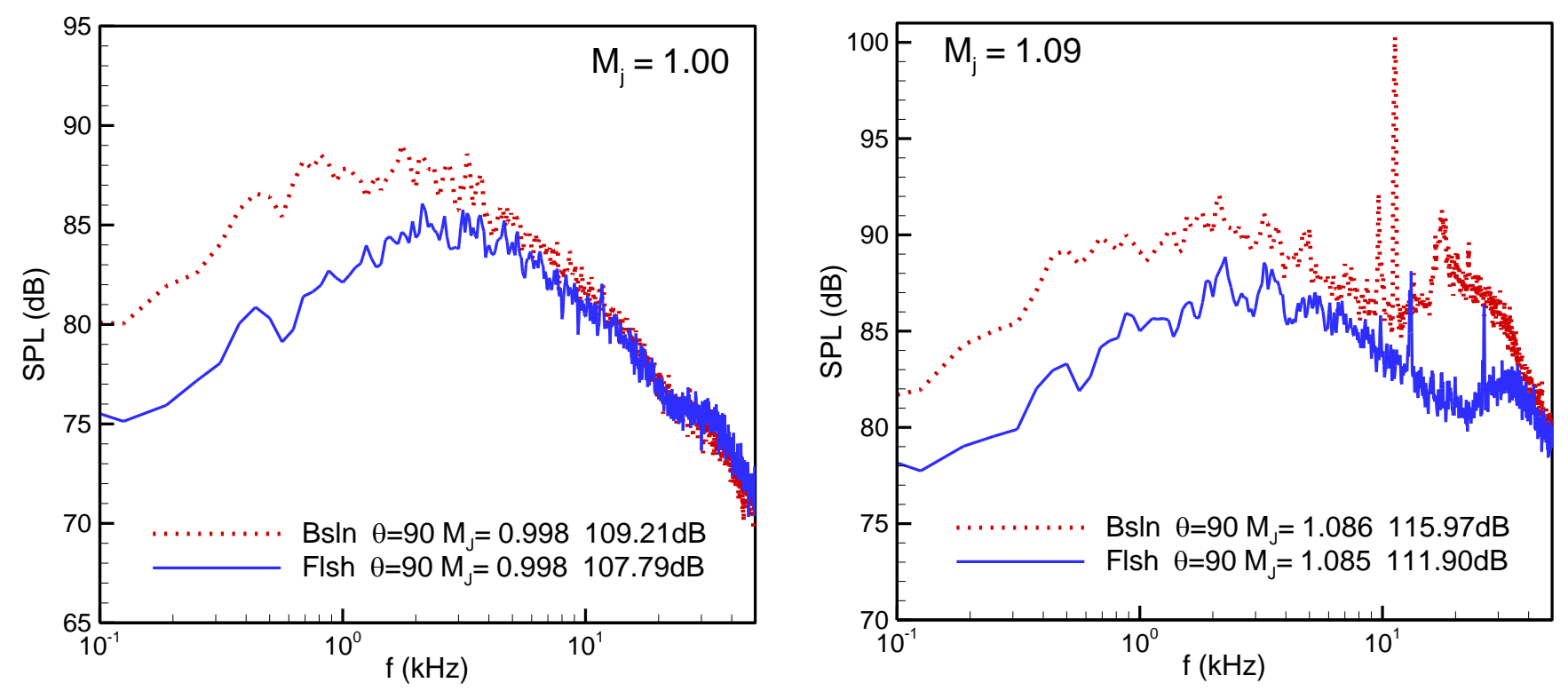


\section{SPL Spectra comparison: Flsh vs other cases at $M_{j}=1$}
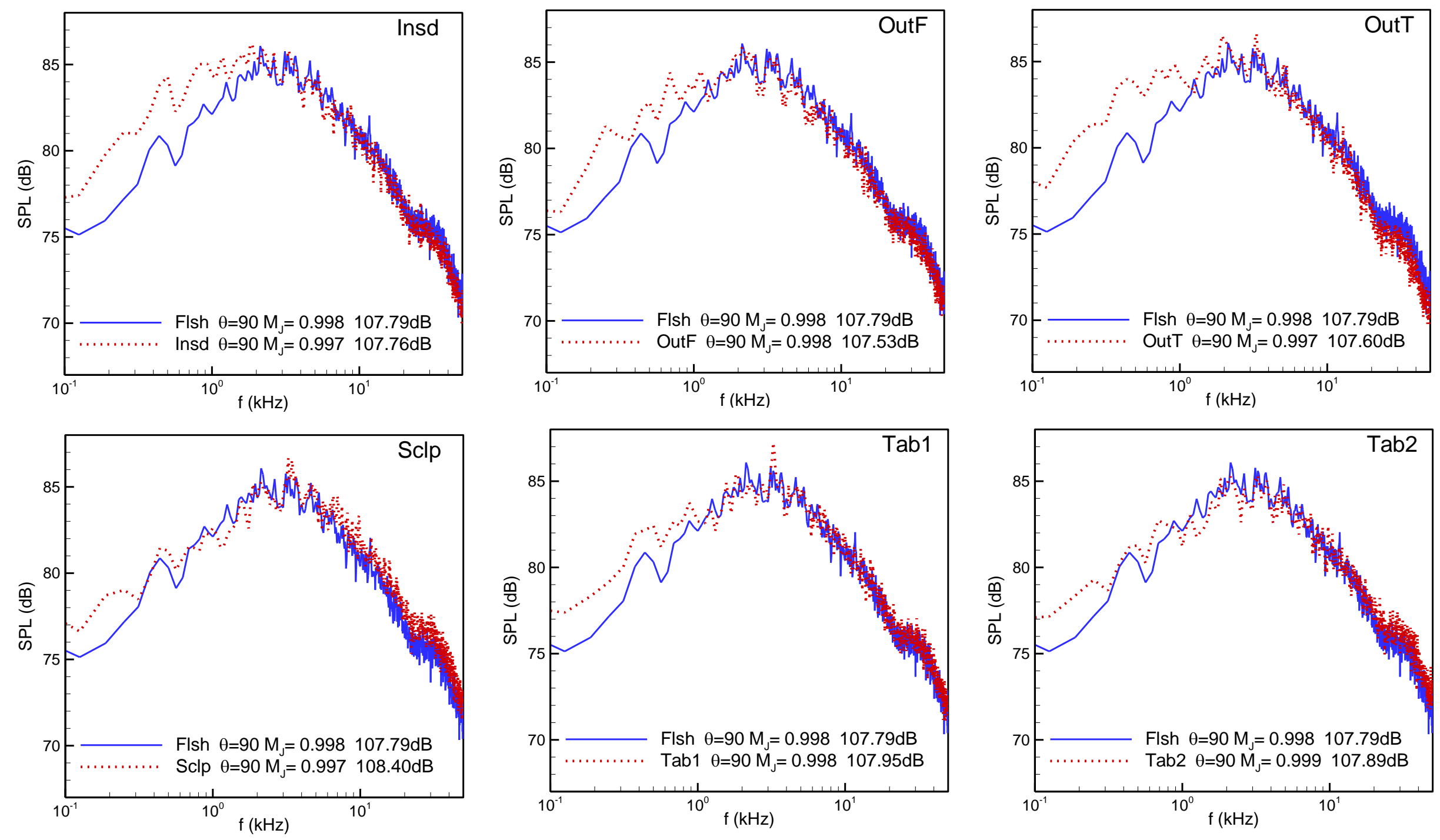

Maximum noise reduction with Flsh case. Sclp and Tab cases have comparable result. 


\section{SPL Spectra Flsh vs Baseline at other angular locations; $M_{j}=1$}
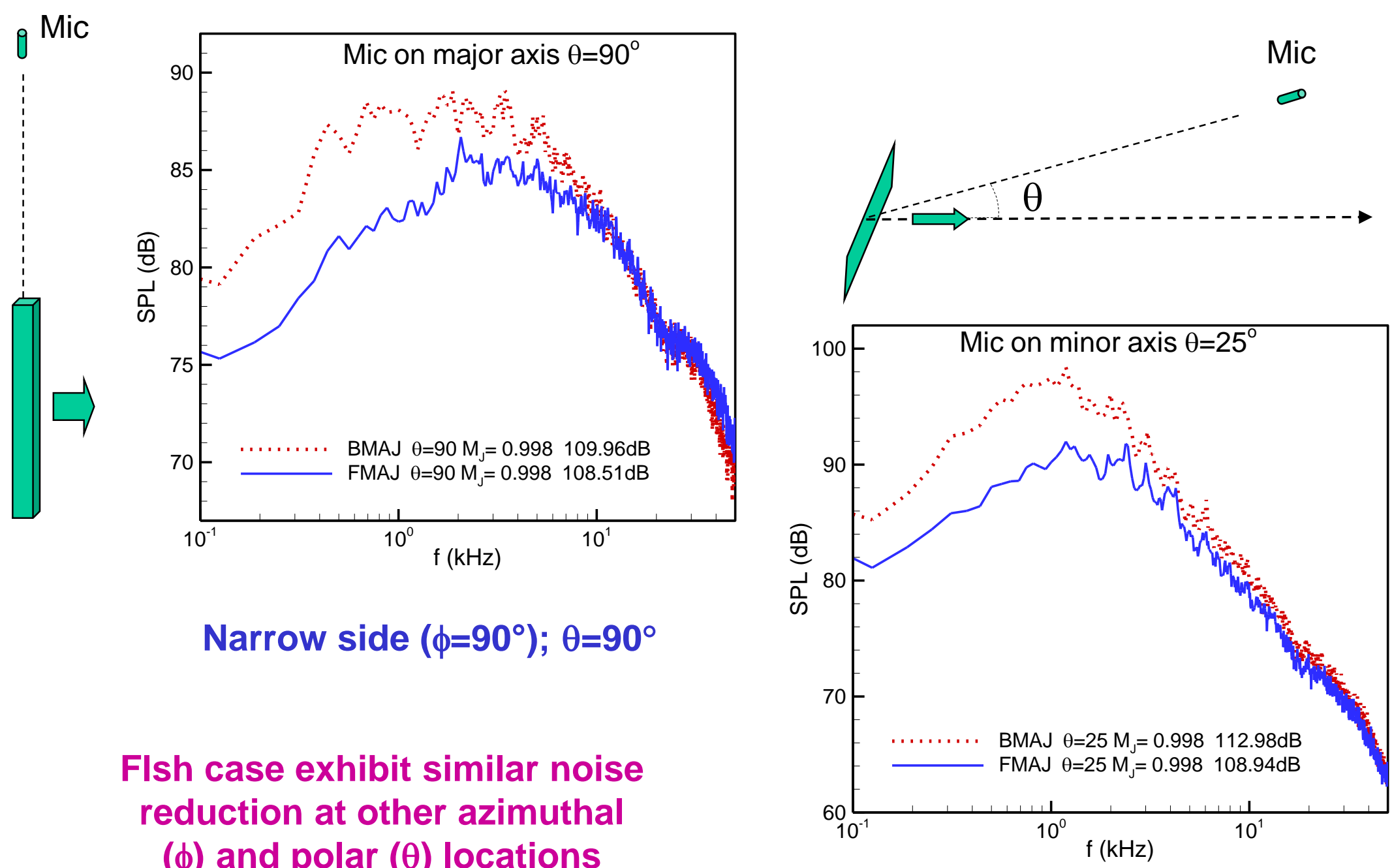

Flsh case exhibit similar noise reduction at other azimuthal

$(\phi)$ and polar $(\theta)$ locations

Narrow side $\left(\phi=90^{\circ}\right) ; \theta=90^{\circ}$

Broad side $\left(\phi=0^{\circ}\right) ; \theta=25^{\circ}$ 


\section{SPL Spectra data measured in the AAPL}

Flsh vs. Baseline cases; $M_{j}=0.99$

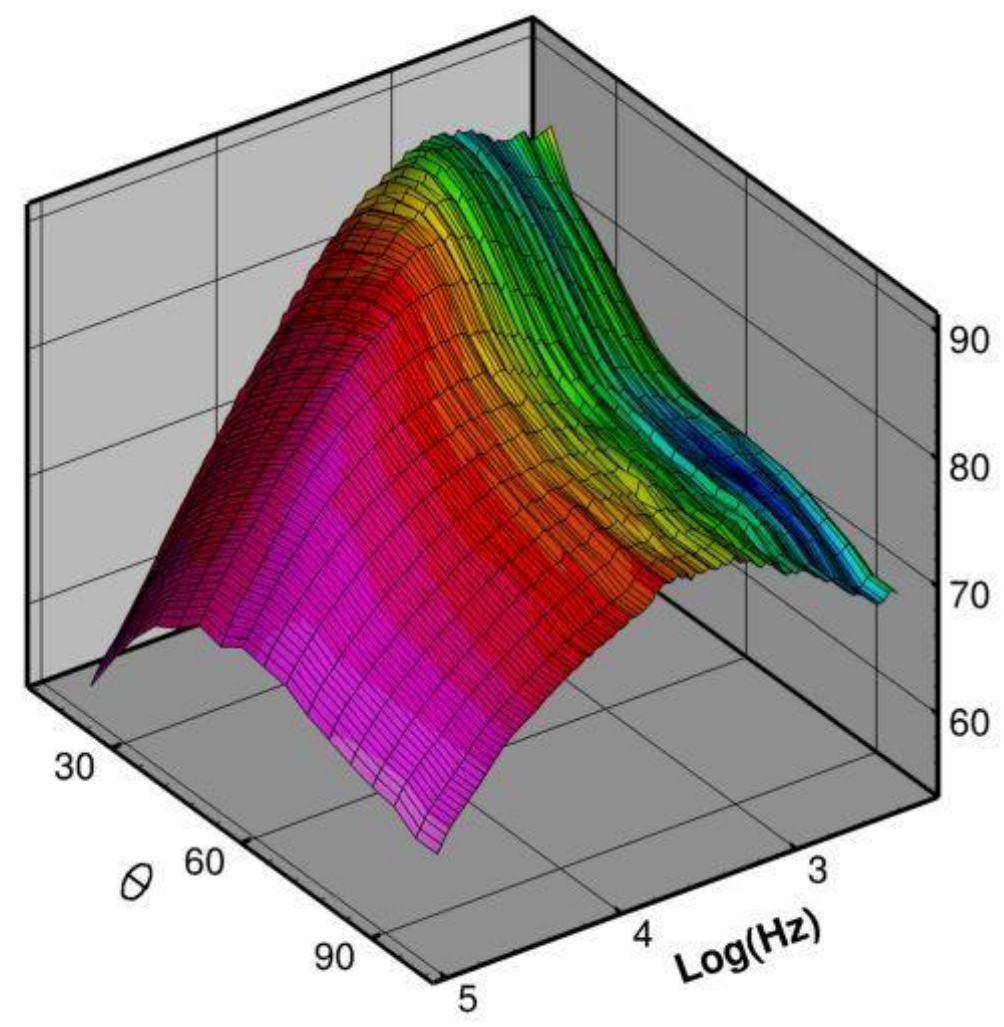

Carpet plot of PSD

Broad side $\left(\phi=0^{\circ}\right)$

$24 \theta$ locations

dPSD (dB)

2
0
-2
-4
-6
-8

$\widehat{\underline{x}}$
क़
Observation in CW17 is confirmed by accurate data taken in the AAPL

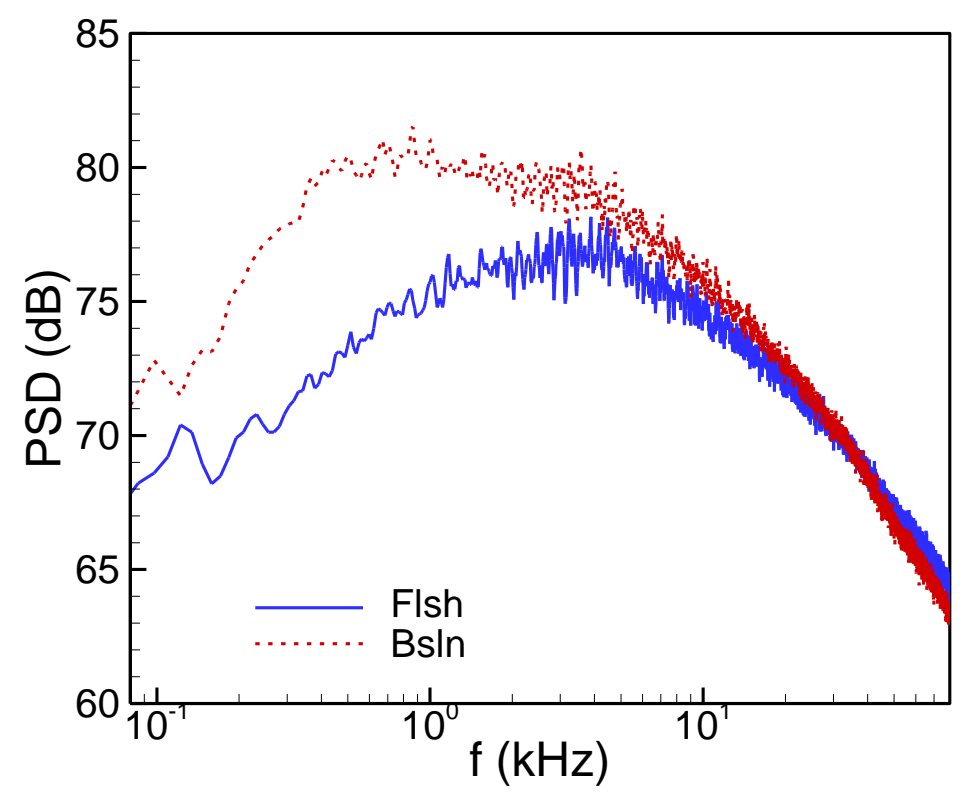

Direct comparison at $\phi=0^{\circ}, \theta=90^{\circ}$ 

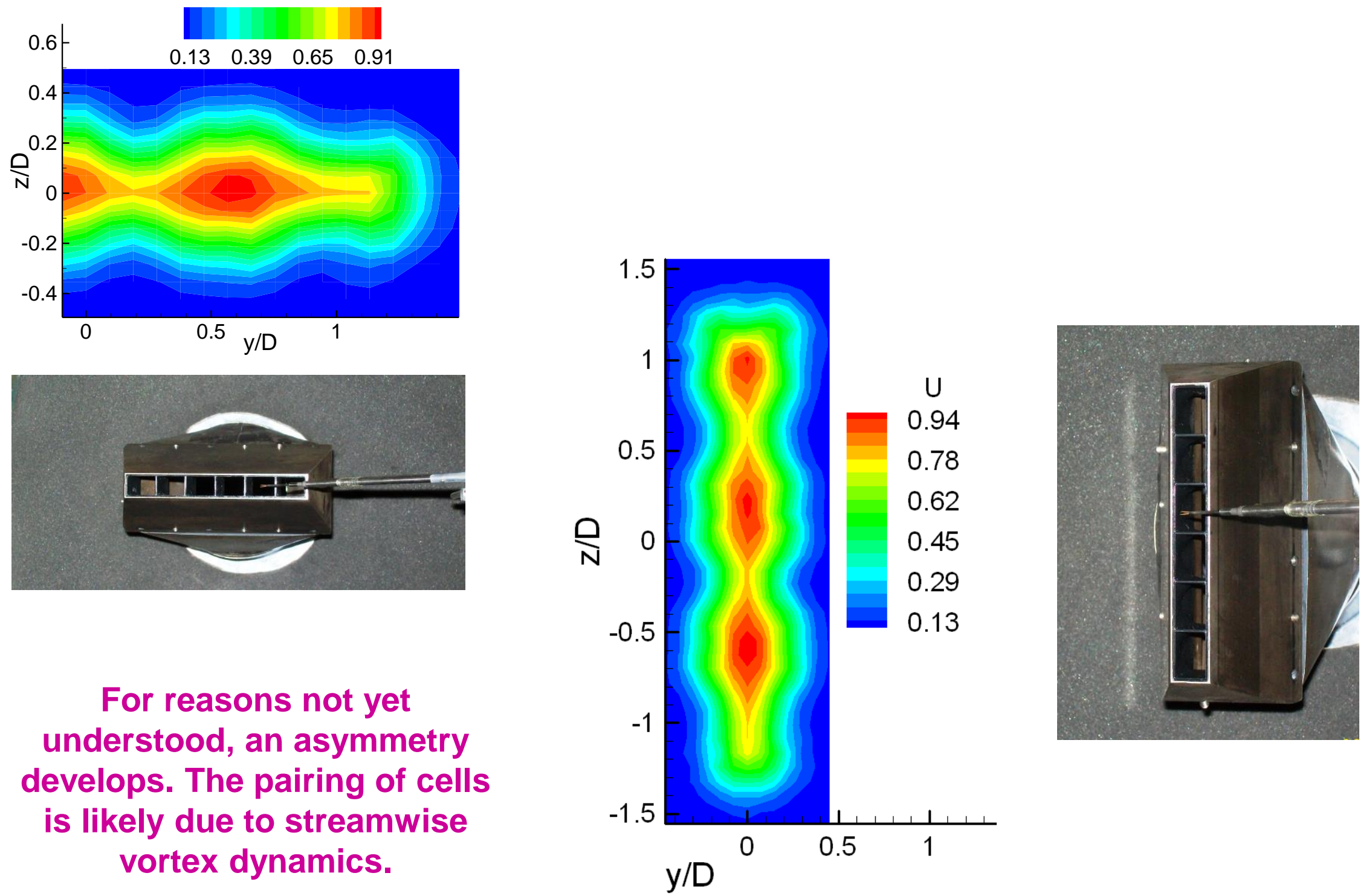

understood, an asymmetry develops. The pairing of cells is likely due to streamwise vortex dynamics. $y / D$ 


\section{$U$ contours at $\mathrm{x} / \mathrm{D}=2 ; M_{j}=0.265$}

\section{6-septa (flush) design I and II and Sclp case}

Design I: rectangular-circular-rectangular passage Design II: rectangular passage thru
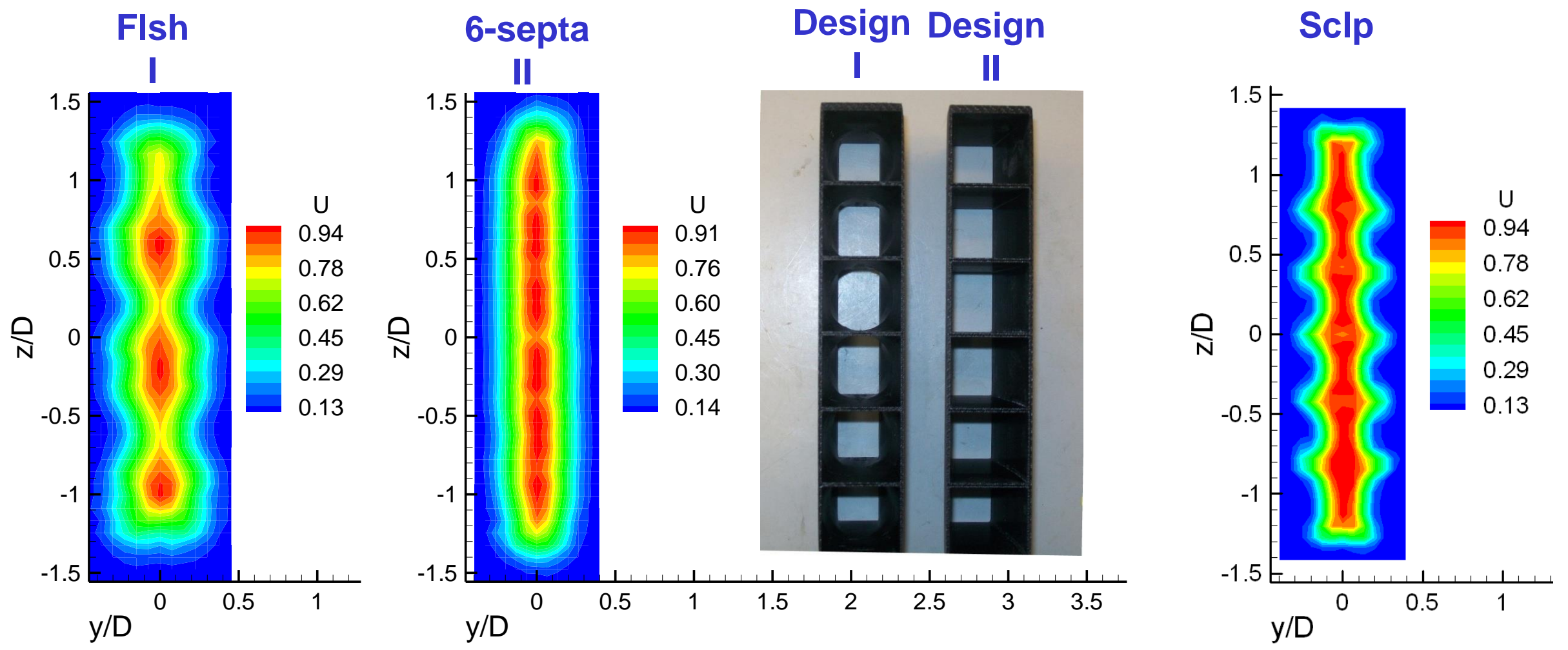

No such pairing with design II case. Note only 5 cells for Sclp case. 


\section{Cross-sectional distributions of $\boldsymbol{U}$ at different $\boldsymbol{X}$}

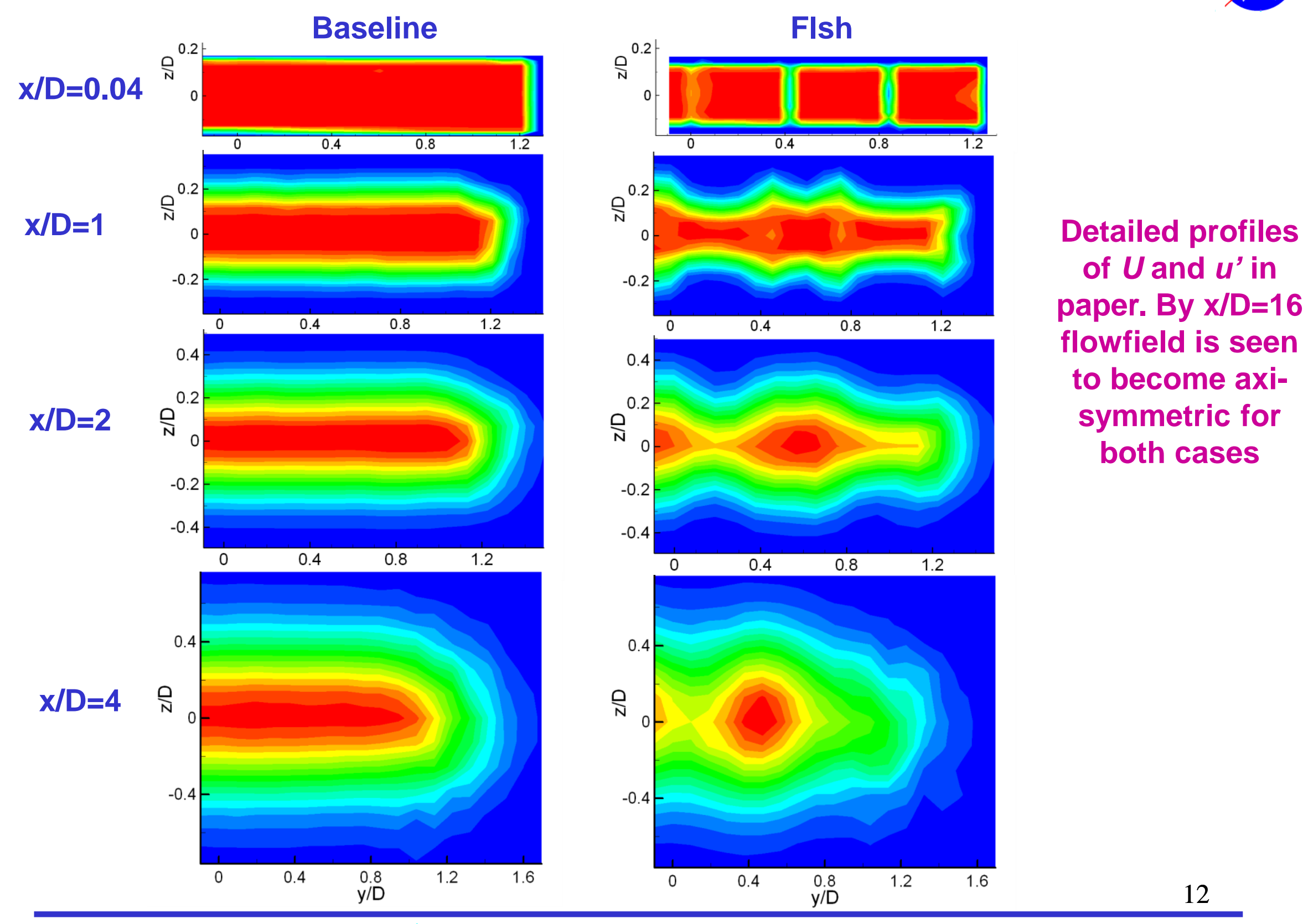




\section{SPL Spectra data for different $\phi ; \theta=90^{\circ}$ Flsh case; $M_{j}=0.52$}

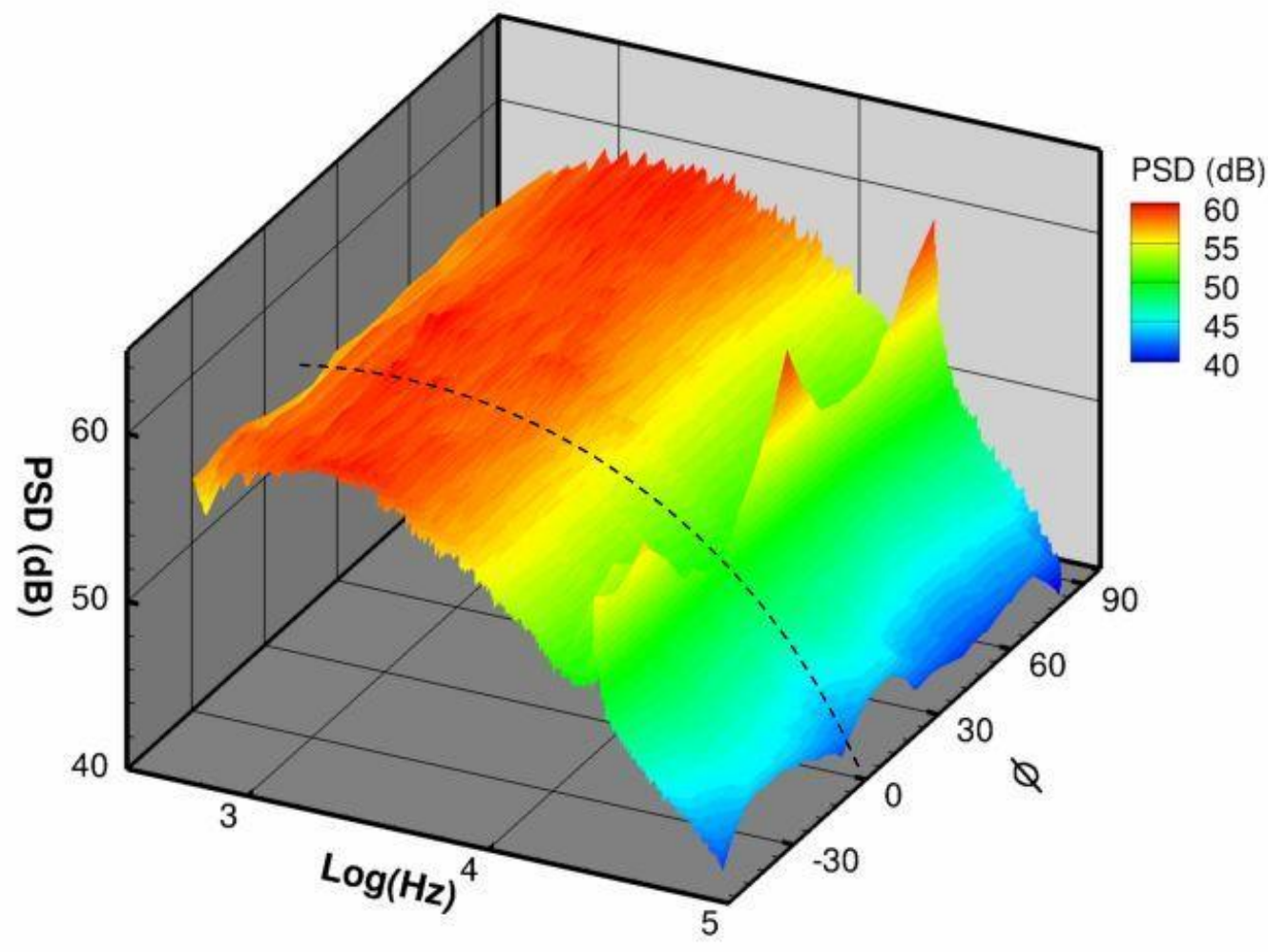

Carpet plot of PSD $24 \phi$ locations AAPL data
Earlier measurement in CW17 $(\phi=0)$ missed the spectral peak. When nozzle turned $90^{\circ}$ peak appeared

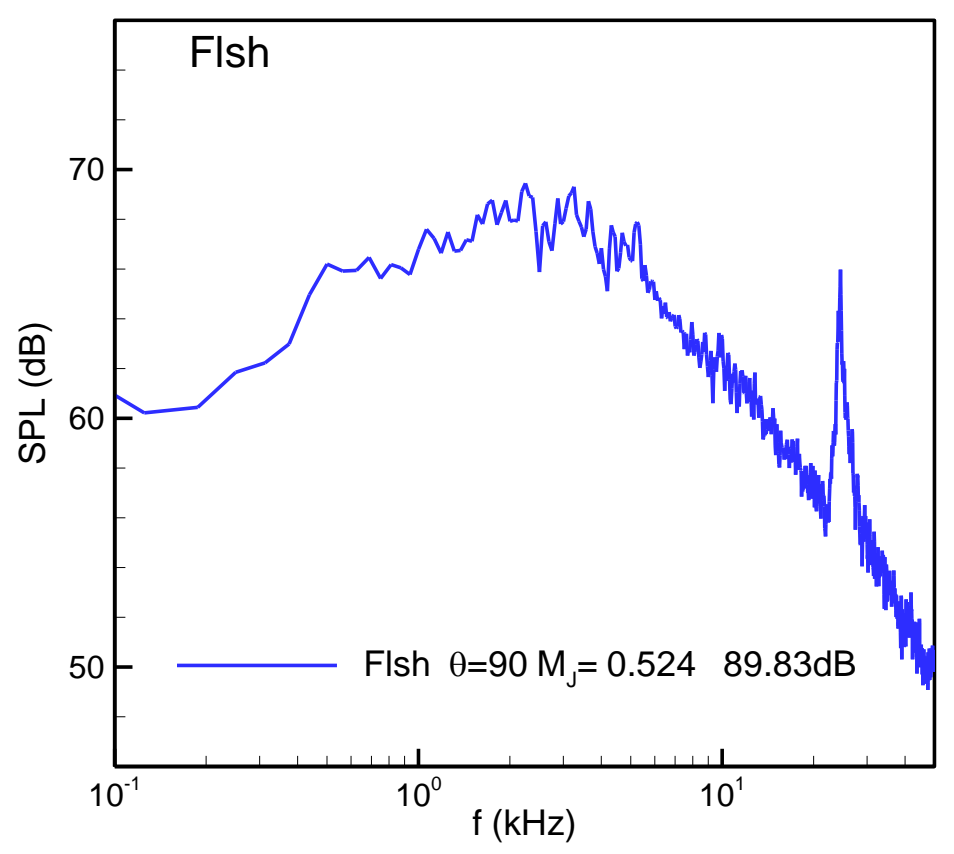

CW17 data on major axis $\left(\phi=90^{\circ}\right)$ 


\section{SPL Spectra at $M_{j}=0.52$ on major axis} for four different inserts compared to Flsh case
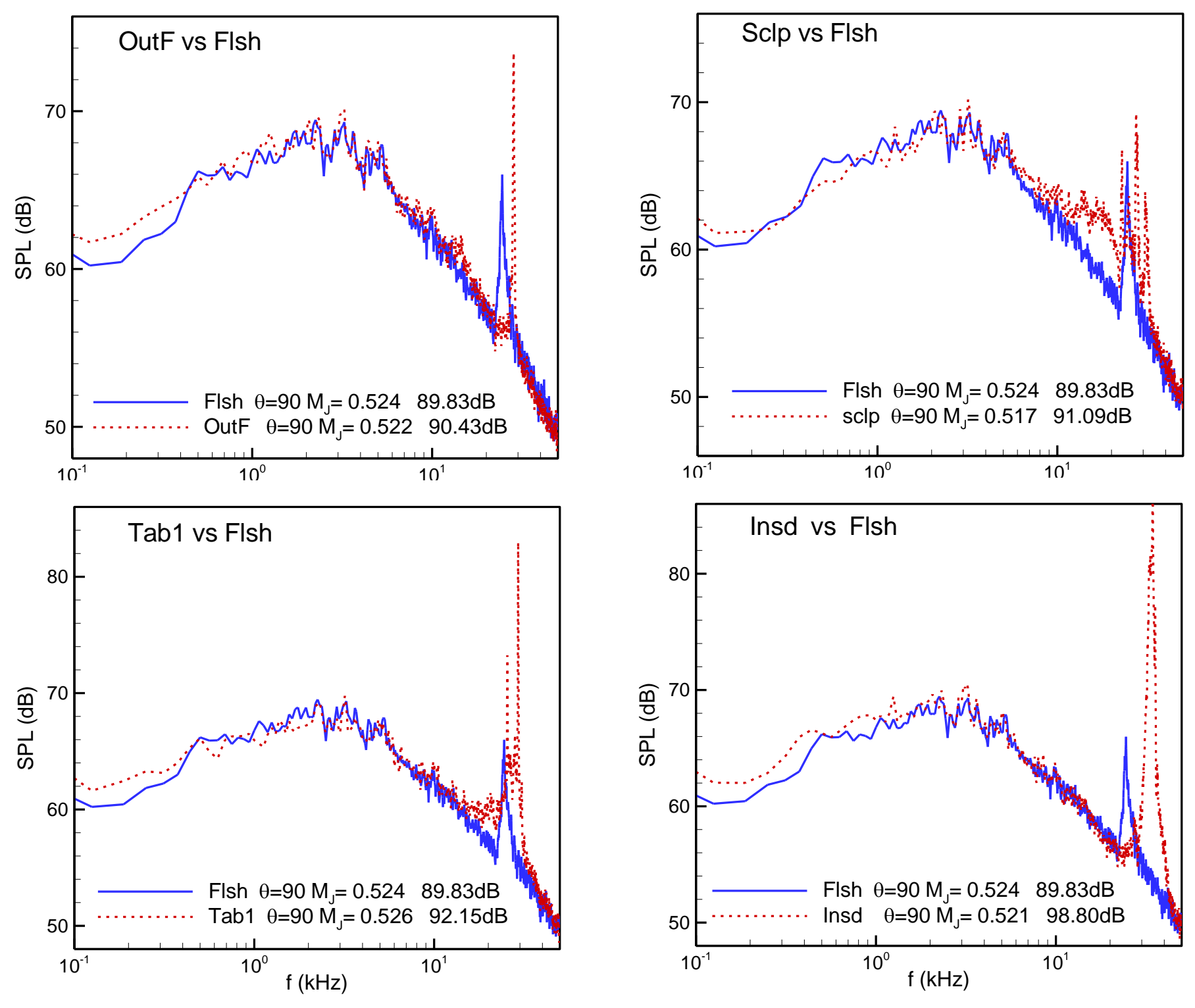

All inserts yield the high-freq spectral peak at low $M_{j:}$. It is most intense with the Insd case 
Strouhal number corresponding to spectral peaks

Data for Flsh and Insd cases

$t=$ trailing edge thickness of partition

(Microphone as well as HW data)
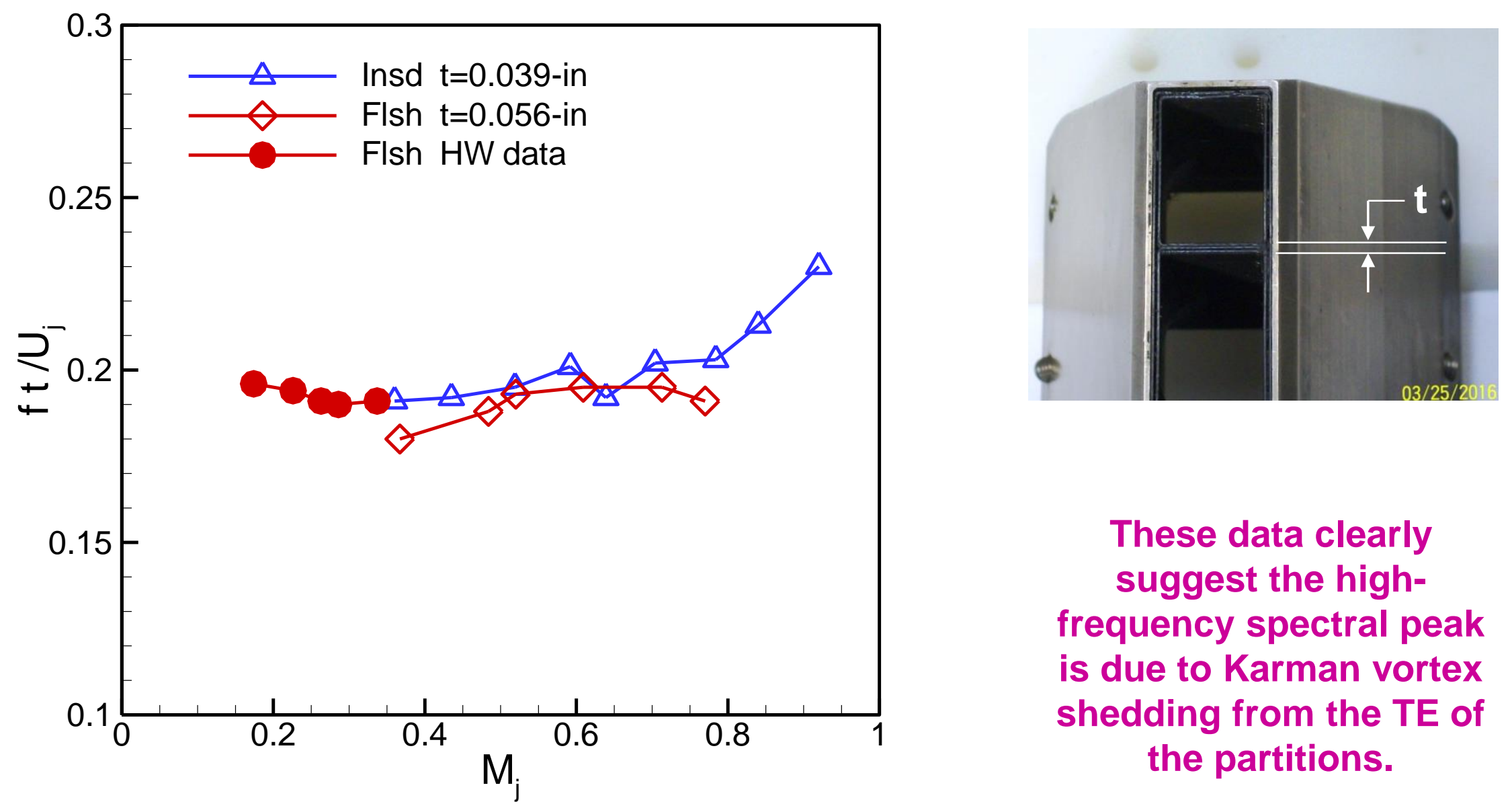

These data clearly suggest the highfrequency spectral peak is due to Karman vortex shedding from the TE of the partitions. 
Baseline

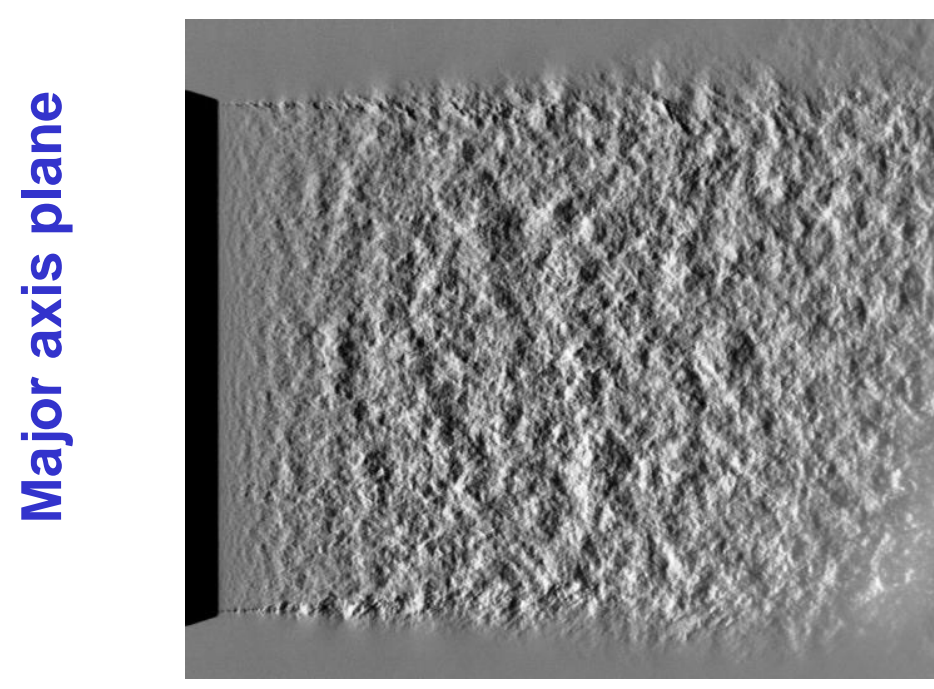

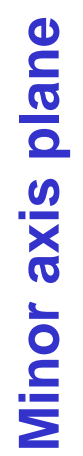

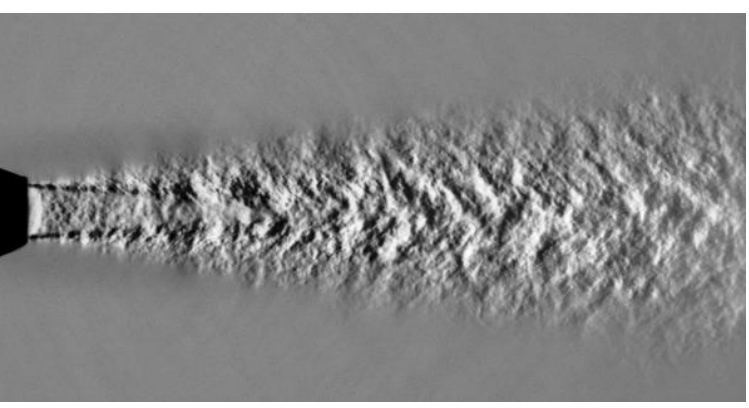

Flsh
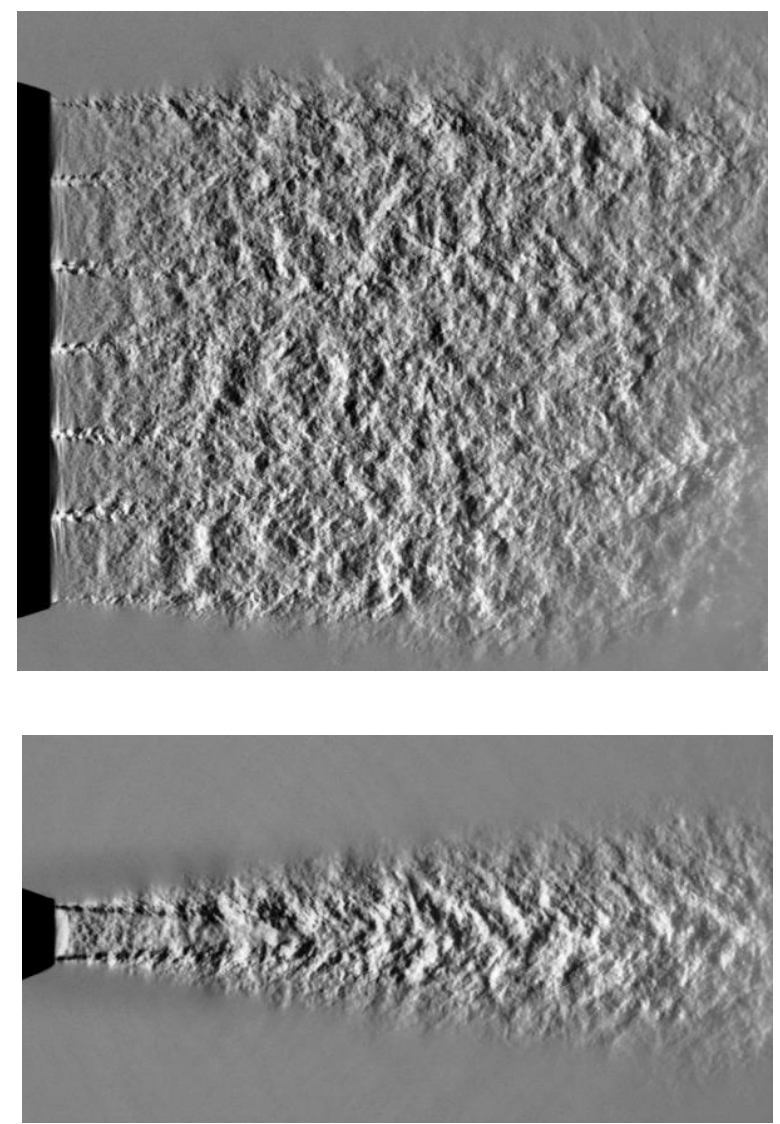

The Flsh case exhibit vortex shedding from the TE of partitions 


\section{Schlieren pictures of flowfield}

Flsh case, major axis plane
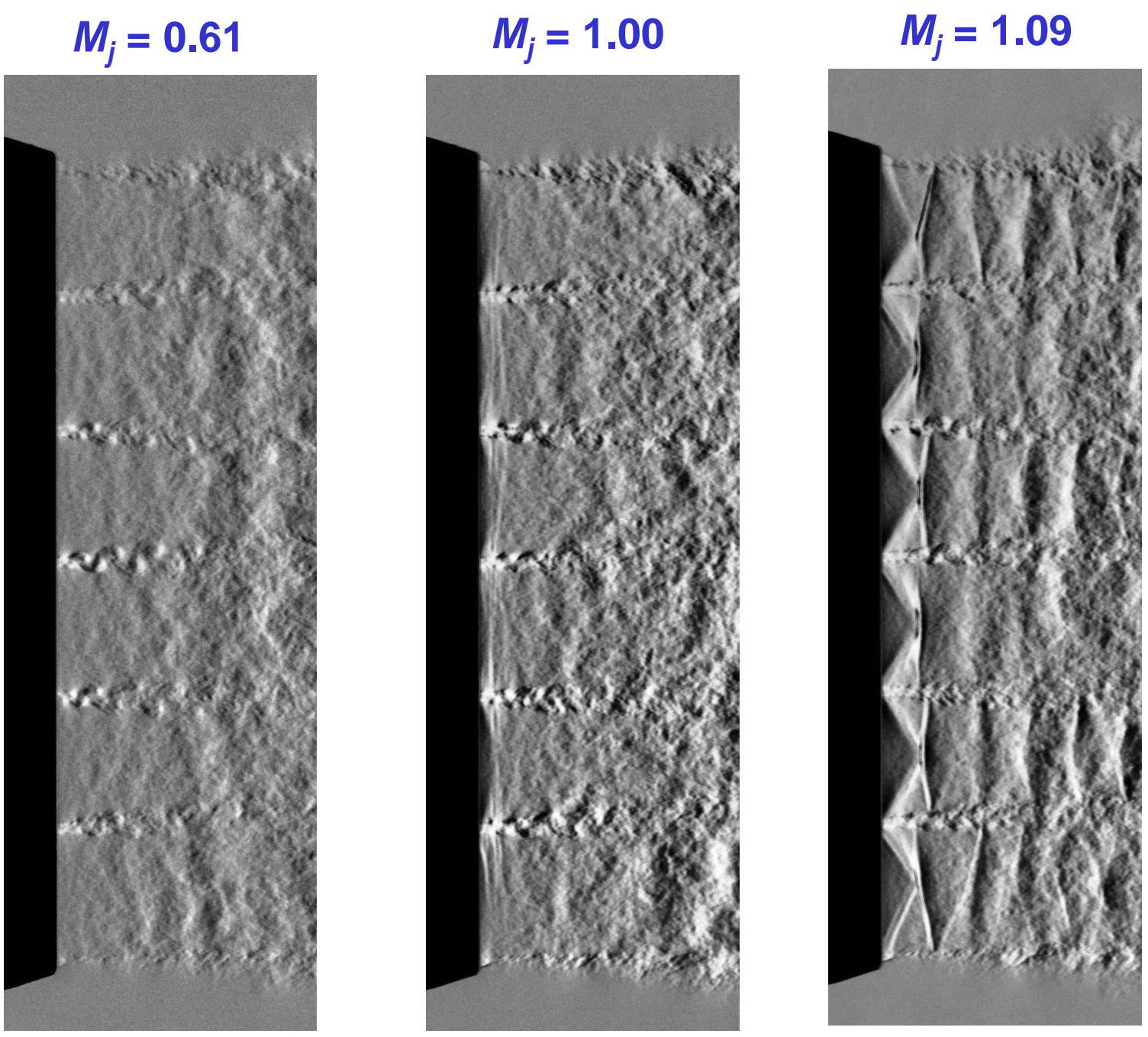

Zoomed-in pictures show the asymmetric vortex shedding that is persistent even at supersonic condition with the presence of shocks 


\section{Conclusions}

Nozzle with septa is quieter than corresponding baseline nozzle.

Cellular flow structure for the septa case (design I) goes through a curious evolution downstream where adjacent cells pair.

Centerline mean velocity exhibit an upstream shift of the jet for the septa case. Turbulence intensity is reduced downstream.

At lower $M_{j}$ a high-frequency tone occurs that is heard prominently on the major axis. It is due to Karmann vortex shedding from the TE of partitions separating the septa. 
Microphone on major axis
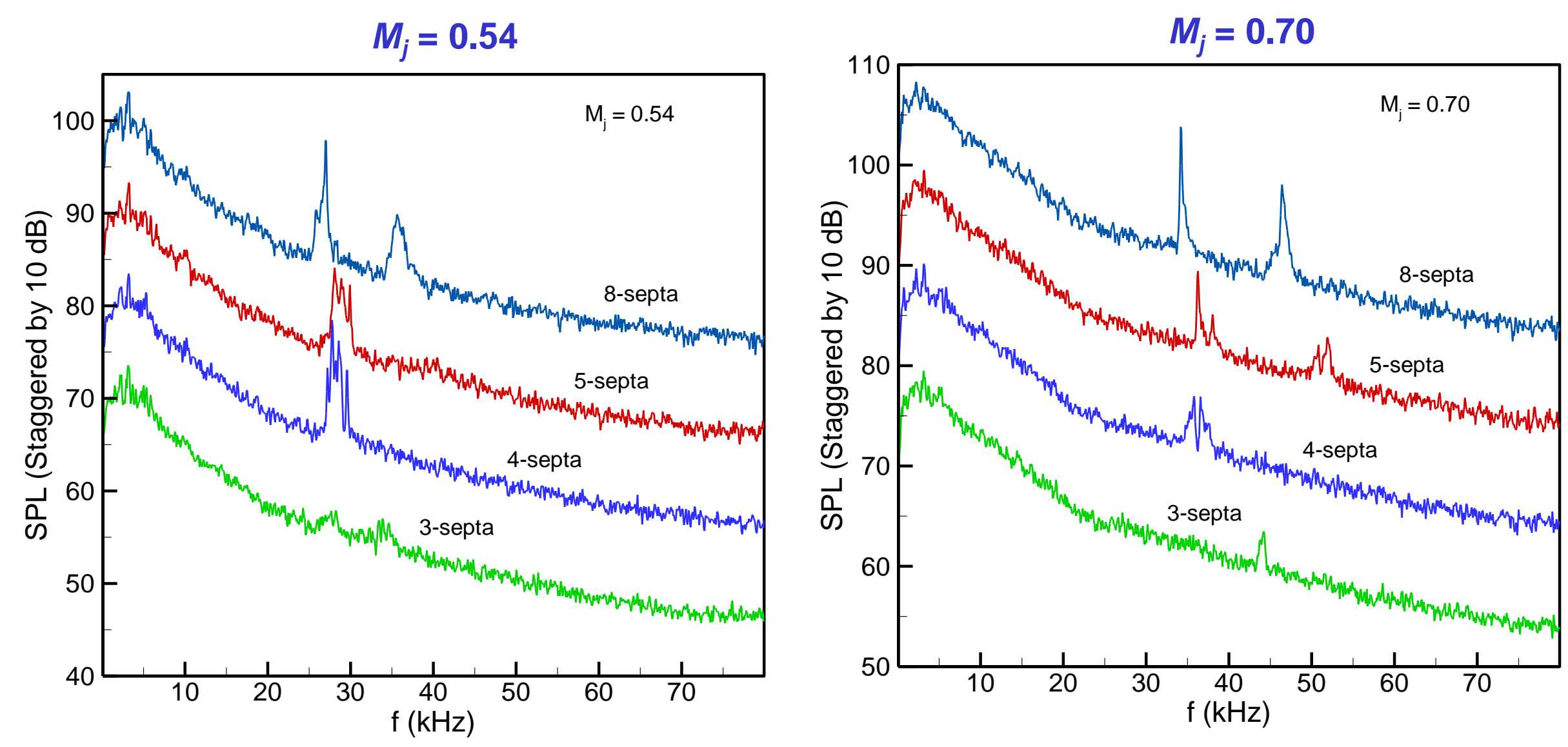

These results demonstrate that the shedding tone intensifies with more number of septa (closer proximity of the partitions). 
NASA Glenn Research Center

Centerline profiles of $U$ and $u^{\prime}$ FIsh vs Baseline cases; $M_{j}=0.265$

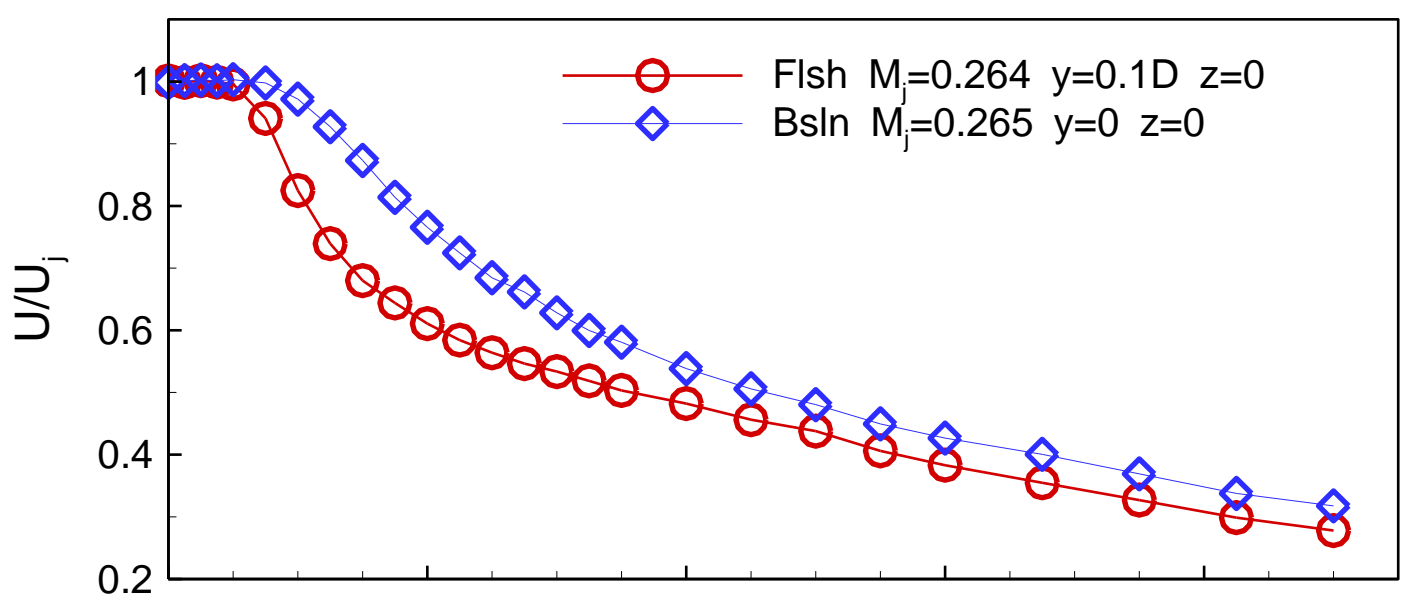

FIsh septa case involves a faster plume decay and lower turbulence downstream

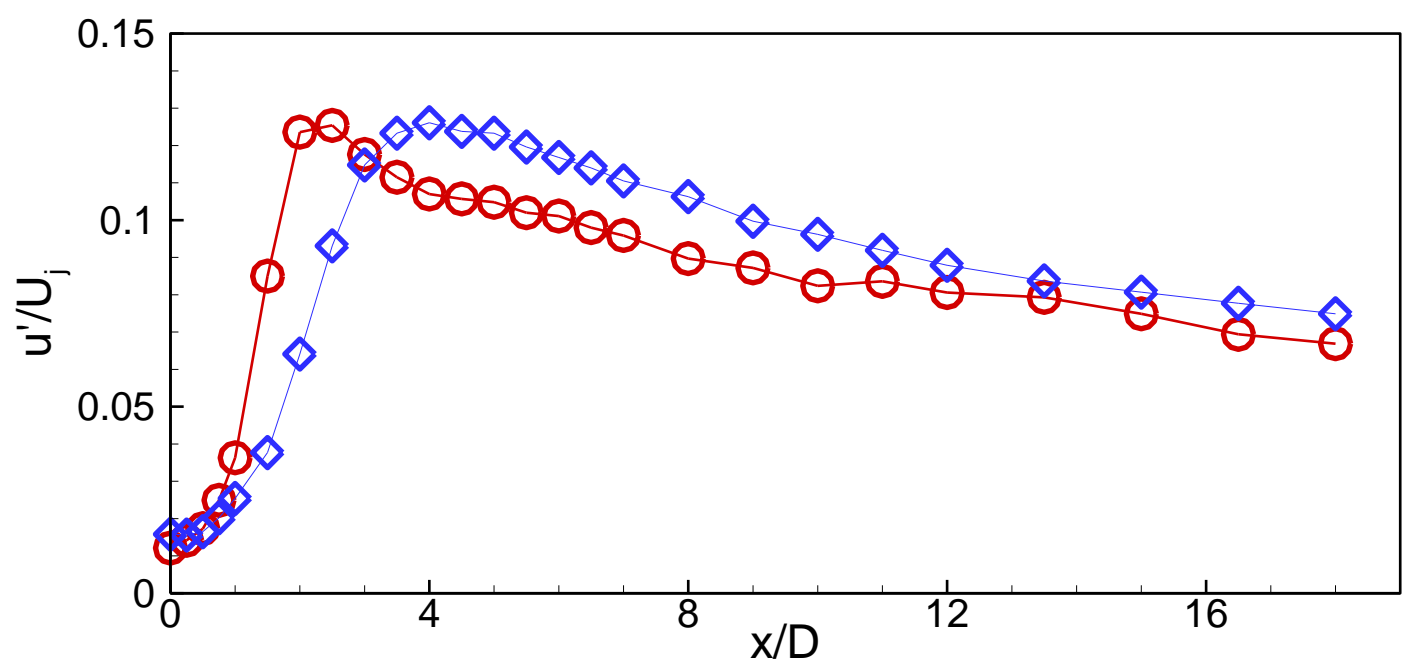

20

22 $2^{\text {nd }}$ AIAA/CEAS Aeroacoustics, Lyon, France, June 1, 2016 Zaman/GRC 